\title{
Impacto de la pandemia Covid -19 en el comportamiento del consumidor en Pachuca, Hidalgo
}

\author{
Covid -19 pandemic Impact on consumer behavior in Pachuca, Hidalgo \\ Alejandra Vega Barrios ${ }^{a}$, Consuelo Goytortúa Coyoli ${ }^{b}$
}

\begin{abstract}
:
Introduction: The impact of the pandemic called COVID -19 has changed consumer behaviour in the municipalities of Pachuca, Hidalgo; Therefore, it is important to consider the effects that have occurred in the establishments from the measures taken to stop the infections. Methods: A bibliographic review was carried out using secondary bibliographic sources. Development: In the city of Pachuca, Hidalgo, the Operative Shield and No Circula Sanitario program is established, which were implemented to minimize COVID-19 infections, so as the pandemic developed and the efforts to contain it, it has been had an impact on consumer behaviour. Conclusion: It is important to ask yourself the question, how has the impact of the pandemic been on consumer behaviour in Pachuca, Hidalgo?
\end{abstract}

Keywords:

Consumer Behavior, Pandemic, COVID -19

\section{Resumen:}

Introducción: El impacto de la pandemia denominada COVID -19 ha cambiado el comportamiento del consumidor en los municipios de Pachuca, Hidalgo; por lo que es importante considerar los efectos que se han presentado en los establecimientos a partir de las medidas tomadas para detener los contagios. Métodos: Se realizó una revisión bibliográfica empleando fuentes secundarias de carácter bibliográfico. Desarrollo: En la ciudad de Pachuca, Hidalgo se establece el programa Operativo Escudo y No Circula Sanitario, los cuales fueron implementados para minimizar los contagios de COVID -19 por lo que a medida que se desarrolló la pandemia y los esfuerzos para contenerla, se ha tenido impacto efectos en el comportamiento del consumidor. Conclusión: Es importante hacerse la pregunta ¿Cómo ha sido el impacto de la pandemia en el comportamiento del consumidor en Pachuca, Hidalgo?

Palabras Clave:

Comportamiento del Consumidor, Pandemia, COVID -19

\section{Introducción}

En el estado de Hidalgo a mediados del mes de marzo de 2020, se confirmaron de forma oficial los primeros dos casos, de COVID-19, los cuales fueron detectados en los municipios de Pachuca de Soto y Mineral de la Reforma. El 12 de mayo el estado entró a la fase 3, una fase de "contagio acelerado". El Sistema de color de semáforo fue anunciado por el Gobierno de México, consta de cuatro colores (verde, amarillo, naranja y rojo) que representan la gravedad de la pandemia en cada estado. Hasta el 21 de junio el estado de Hidalgo se mantenía en color rojo. Al momento que esta revisión fue finalizada el 30 de diciembre del 2020, el municipio de Pachuca de Soto supera los 5,200 casos confirmados acumulados. [1] [2] [3]

Es por ello por lo que, a partir de esta información, es importante conocer ¿Cuáles ha sido el impacto de la pandemia del COVID-19 en el comportamiento del consumidor en Pachuca, Hidalgo? ¿Cómo se han

\footnotetext{
a Corresponding author, Universidad Autónoma del Estado de Hidalgo, Programa educativo de mercadotecnia, https://orcid.org/0000-0002-2368-7939,Email: vbarrios@uaeh.edu.mx 
adaptado los consumidores a partir del programa Operativo Escudo y el No Circula Sanitario?

Los impactos más importantes a nivel económico ha sido el sector de la construcción en Hidalgo en el primer trimestre del 2020, teniendo una baja en su actividad productiva en $8.2 \%$ comparada con el 2019, (Hernández, E, 2020) De acuerdo con la Cámara Nacional de Comercio, Servicios y Turismo de Pachuca (CanacoServytur) se estima que la pandemia ha provocado la caída en ventas en un $80 \%$. (Hernández, 2020). La pandemia ocurrió en uno de los periodos con mayor afluencia de visitantes, por lo que más de 13 mil empleos directos fueron afectados. [5] De acuerdo con el Instituto Mexicano del Seguro Social (IMSS) de marzo a mayo se perdieron 10, 929 empleos formales [7].

Como resultado de la pandemia de COVID-19, el mundo ha cambiado y el municipio de Pachuca, Hidalgo también. A nueve meses de la pandemia, todavía no existe un posible periodo post pandemia, cada vez se habla de más contagios y mayores problemas sociales y económicos. Sin embargo, los consumidores para sobre vivir están llevando a cabo cambios en su comportamiento de consumo de productos y servicios a partir de las limitaciones con los programas Operación Escudo y No circula Sanitario.

\section{Métodos}

Se realizó una revisión bibliográfica con búsqueda de fuentes secundarias, la revisión bibliográfica se ha basado en estudios recientes sobre el comportamiento del consumidor, La interrogante para esta revisión es: ¿Cómo ha sido el impacto de la pandemia en el comportamiento del consumidor en Pachuca, Hidalgo? Llevando a un acercamiento para entender las decisiones de consumo que se toman en tiempos de pandemia y su impacto en el comportamiento del consumidor en Pachuca, Hidalgo

\section{Desarrollo}

\section{Comportamiento del consumidor}

Schiffman (2010) definió el comportamiento del consumidor como "la conducta que los consumidores exhiben al buscar, comprar, utilizar, evaluar y desechar productos y servicios que ellos esperan que satisfagan sus necesidades". (p.22) El comportamiento del consumidor puede definirse como un proceso que comprende ciertas características particulares, siendo una de ellas, constar de numerosas actividades en la que se encuentra involucrado el consumidor, abarcando incluso las actividades que preceden, acompañan y siguen a las decisiones de compra [9]. De acuerdo con Torreblanca Diaz et al (2012) El conjunto de actividades que engloba el comportamiento del consumidor están implicados procesos mentales y emocionales, además de acciones físicas. Se basa, por tanto, en un proceso psicológico en el que están incluidas distintas y complejas actividades mentales, cogniciones y emociones que vienen motivadas e influidas por factores fisiológicos, económicos, sociales, ambientales y psicológicos, externos e internos al propio individuo y que, en consecuencia, pueden variar enormemente de una persona a otra o de un grupo de personas a otro y que se expresa a través de una notable variedad de conductas.

\section{Teorías del Comportamiento del consumidor}

\section{La teoría de la utilidad}

La teoría del comportamiento del consumidor explica el concepto de "utilidad" a partir de las necesidades del hombre, entendiendo a estas como la sensación de carencia. Las necesidades al hombre son ilimitadas en cuanto a su cantidad, pero limitadas en cuanto a su capacidad. Según (Brue y Grant, 2009), esta teoría aborda todos los incentivos para la acción y medirse a través de unidades monetarias, siendo el beneficio resultante de la toma de una decisión que acarreó consigo una acción. Indistintamente del bien o servicio que una institución, $u$ organización. En tanto que analiza el comportamiento de los consumidores en base a elecciones y preferencias, mostrando cómo las personas organizan su salario.

\section{Enfoque Cardinalista}

En este enfoque Hall y Lieberman (2012) definen a la utilidad como el nivel de satisfacción que se alcanza al consumir un bien y se mide en útiles. Esta teoría es la más antigua, es importante por el significado de la distinción que hace entre utilidad marginal que representa la utilidad que se obtiene al incorporar una unidad adicional del bien

\section{Teoría neoclásica del consumidor}

Esta teoría plantea que un consumidor posee un presupuesto que puede ser gastado en una diversidad de productos disponibles en el mercado y que la distribución de ese presupuesto será realizada de acuerdo con las preferencias del comprador como menciona Bryant (1990) "la demanda de bienes y servicios es el resultado de la interacción entre sus preferencias hogar y sus posibilidades, estas últimas representadas por la restricción presupuesto" (p.17).

\section{Teoría psicológico-social}

De acuerdo con Kotler y Keller (2016) "el comportamiento de los consumidores no solo está determinado por variables económicas sino también por aspectos psicológicos tales como la personalidad, los deseos y las necesidades". (p.76) por lo que también se toma de referencia factores tanto externos [cultuales, sociales] como internos [personales, psicológicos] para la decisión del consumo. 
Teoría de la Motivación Humana

Maslow (1943) citado en Colvin y Rutland (2008) propone la jerarquía de necesidades y factores que motivan a las personas que buscan satisfacer primero las necesidades básicas para luego pasar a las necesidades fisiológicas, necesidades de seguridad, autoestima, reconocimiento y autorrealización.

\section{Teoría conductual de aprendizaje}

Este enfoque de acuerdo con Hilgard y Bower (1980) plantea que existe un proceso de aprendizaje a través de estímulos cuyas respuestas modifican y condicionan el comportamiento del consumidor.

\section{Teoría del Condicionamiento Operante}

Skinner (1975) definió el condicionamiento operante, como un proceso por el cual el comportamiento es el resultado favorable a partir de un estímulo y, por eso, tiene la probabilidad de que vuelva a repetirse.

\section{Tendencias del comportamiento del consumidor en la nueva normalidad en Pachuca Hidalgo}

La llegada de las nuevas tecnologías de la información (TIC) ha provocado un cambio en el comportamiento del consumidor. Nos encontramos ante un nuevo consumidor que está mucho más informado y que gracias a Internet y las redes sociales puede conocer multitud de opiniones sobre los productos que quiere comprar y aportar la suya (Barrullas, 2016). Por otra parte, según Sheth, (2020a) los hábitos de consumo se ven alterados por cambios en los contextos, social, tecnológico y medidas como reglas y regulaciones relacionadas con espacios públicos y compartidos, así como eventos menos predecibles como terremotos, huracanes, pandemias globales como el caso de COVID-19.

\section{Compras en línea}

En entrevista para el periódico el Economista, López (2021), detalla que Pierre-Claude Blaise, director general de la Asociación Mexicana de Venta Online (AMVO), expresa los principales cambios los hábitos de los consumidores debido a las restricciones sanitarias. Siendo que el $56 \%$ de los consumidores realizaran compras en línea. Los productos de mayor interés para comprar en línea de acuerdo con el estudio de AMVO al mes de enero del 2021, fue la comida a domicilio con un $44 \%$ y Tecnología con un $38 \%$, seguido de super mercado en un $29 \%$. Por otra parte, es importante visualizar el perfil demográfico del comprador digital, donde resalta el segmento femenino con una edad promedio de 45 años y con Niveles socioeconómicos (NSE) AB C+, las preferencias en categorías de servicios más compradas son servicios de suscripción con un $83 \%$, telefonía y servicios bancarios con un $78 \%$, a nivel nacional. En la ciudad de Pachuca, Hidalgo se ha iniciado con la virtualización de comercios, mercancías, pagos a distancia y compras en línea, con lo que están haciendo frente a la pandemia del Covid-19 debido a las medidas sanitarias impuestas por el gobierno estatal.

\section{Entregas a domicilio}

Las aplicaciones de entrega de comida a domicilio virtuales de acuerdo con Ruiz (2020) citado en Vega et al (2021) como Uber Eats, Rappi, Sin Delantal, entre otras, que han permitido y facilitado la continuidad en la operación de restaurantera bajo condiciones estrictas de higiene. El segmento de la población que no se acercaba a este tipo de servicios en línea, ya sea por miedo al robo de datos bancarios o por la falta de conocimiento, durante esta etapa de aislamiento social se ha convertido en aliados para llevar comida, medicamentos y mercancía en general; en la ciudad de Pachuca, se ha implementado que no solo son aplicaciones digitales quienes hacen entregas a domicilio, sino también particulares que han perdido su empleo y buscan una forma de subsistir mediante la entrega de productos a domicilio.

\section{Entretenimiento en línea}

El estudio de la agencia MRM Madrid (2020), citado en Vega et al (2021) identifica el aumento de consumo de contenidos registrado y en las plataformas de streaming como Netflix, HBO o Movistar; de igual forma se observa el aumento del uso de los videojuegos por la generación $Z$ y las plataformas de streaming especializadas, como Twitch han incrementado su tasa de interés, así como el uso de plataformas de música. De acuerdo con Comscore (2020) México es de los países que más consume contenido a través de aplicaciones a través de sus smartphones (teléfonos inteligentes). Debido al impacto del COVID-19, algunas aplicaciones crecieron como contenidos infantiles, política, gobierno, social media, deportes/outdoor y fitness; mientras que deportes, real state, automotor y viajes disminuyeron su porcentaje de interés en las audiencias. En Pachuca, también se incremento el consumo de plataformas como Netflix, ya que al ser implementada la contingencia y el programa no circula sanitario se tuvo mayor acceso.

\section{Educación en línea}

A partir de una pandemia, es como la tecnología que ya se encontraba en nuestras vidas, se ha establecido como parte fundamental, llevándonos a la adquisición de la educación en línea, actualmente con acceso a través de aplicaciones que si bien ya existían se potencializaron durante la pandemia, como: Zoom, Meet, Webex, Teens 
para los aspectos académicos en todos los niveles, así como el uso de servicio web educativo como lo es Classroom de la suite de Google, para el apoyo a la educación, facilitando a los estudiantes los contenidos, la administración del curso por parte de los docentes y el espacio para interactuar entre los alumnos. En la ciudad, cada una de las escuelas ha tratado de llevar a los participantes de los niveles educativos, bajo un seguimiento en plataformas propias de las instituciones o en plataformas gratuitas con la intención de dar educación a las personas en medio de la crisis sanitaria.

\section{Programa Operación Escudo}

El 10 de marzo de 2020, aún sin haber casos de personas infectadas por el nuevo virus, el Gobierno del Estado de Hidalgo, a través de su titular el Lic. Omar Fayad Meneses, gobernador constitucional presenta un plan de contingencia en el cual resaltan la difusión sobre la prevención, detección y tratamiento del coronavirus a través de la prensa, el radio, la televisión y las redes sociales; la coordinación operativa de todas las unidades de salud estatales, la revisión y ampliación de la reserva estratégica, los acuerdos de colaboración, los simulacros de adecuación y la instalación de un hospital inflable. [23]

Para el 16 de marzo del mismo año, se da a conocer el Operativo Escudo, por un Hidalgo Sano y Seguro, , ante la inminente llegada de contagios de covid-19 a México, esto con el objetivo de disminuir la propagación del virus SARS-CoV-2. El principal objetivo era y es detener una posible crisis de salud y económica en el Estado de Hidalgo. [24]

\section{Estrategia Hoy No circula sanitario}

Una de las estrategias para reducir los contagios por COVID-19 implementadas por el gobierno del Estado de Hidalgo es el programa para reducir la movilidad denominado Hoy no Circula Sanitario el cual fue anunciado el 2 de mayo de 2020 por las autoridades de Gobierno del Hidalgo, implementándolo obligatoriamente a partir del 4 de mayo del mismo año. Esta medida aplica a los vehículos que tengan placas del estado de Hidalgo y de otras entidades $y$, además de respetar las fechas de circulación de acuerdo con el número de placa, también deben acatar que los vehículos compactos no podrán circular con más de dos personas. [25]

\section{Conclusiones}

Después de revisar la literatura en relación con el comportamiento del consumidor y conocer las tendencias en la nueva normalidad, podemos contestar las preguntas que nos realizamos al principio de esta investigación ¿Cuáles ha sido el impacto de la pandemia del COVID-19 en el comportamiento del consumidor en Pachuca,
Hidalgo? Hemos observado que la pandemia, ha dejado un impacto importante en los habitantes de la ciudad, así como ha cambiado la realidad que se tenía, las tecnologías de la información han ayudado a hacer los cambios más rápidos, sin embargo vemos que la teoría psicológico - social, se ha impuesto pues no solo el comportamiento de los consumidores están definidos por las variables económicas que en estos momentos se encuentran con poco crecimiento, de tal forma que se requieren los aspectos psicológicos como la personalidad y la referencia de los factores internos y externos para continuar aún en las declaratorias de la pandemia.

Por otra parte, ¿Cómo se han adaptado los consumidores a partir del programa Operativo Escudo y el No Circula Sanitario? En realidad, los consumidores han tenido que cambiar sus hábitos de consumo, como ha sido las compras en línea, que, a pesar de ser un esquema de hace algún tiempo, no había sido tan explotado como se ha realizado por la pandemia; lo mismo sucede con las entregas a domicilio, ya que han aparecido empresas que ofertan el servicio con un buen costo y con las medidas sanitarias solicitadas por las autoridades y por las familias que los contratan.

En cuanto a la Educación en línea, no era una categoría esperada por los padres de familia, ya que, de forma tradicional, los estudiantes recibían clases en un formato presencial, y en el inicio de la pandemia, no se tenía preparación para la impartición de la asignatura en la modalidad virtual, como tampoco el alumno estaba acostumbrado a vivir este tipo de experiencia, aislado en su domicilio, utilizando herramientas tanto sincrónicas como asincrónicas para seguir con su preparación, vale la pena mencionar que para algunos ha sido sencillo, pero para la mayoría ha sido muy complicado el método de estudio y no contar con las facilidades de las herramientas digitales, ni el espacio destinado para tener una clase.

Al revisar el entretenimiento en línea, quizá haya sido, el aumento de la visualización de las plataformas, que, aunque ya existían no tenían un consumo tan alto, sin embargo, con el programa Operación Escudo y la estrategia Hoy no circula sanitario, se obligo a la población a estar más tiempo en casa y por ello la búsqueda de nuevas actividades, con lo que se tenía, desde el inicio de la pandemia.

Concluimos que el impacto es altamente significativo, y la adaptación al programa Operación Escudo, que tenía como objetivo detener una crisis tanto económica como de salud, ha sido muy lenta y poco entendida por la población, así como la estrategia Hoy no circula sanitario pues la intención era reducir la movilidad para evitar la 
propagación del virus SARS-CoV-2, no obstante, los datos de los contagios siguen incrementándose.

\section{Referencias}

[1] Reyes, A. (2020). Confirman primeros casos de coronavirus en Hidalgo. Pachuca: Milenio Hidalgo (Milenio Diario)

[2] Montoya, R. (2020). «Hidalgo entra a fase de "contagio acelerado" por Covid-19». Ciudad de México: La Jornada.

[3] Periódico Criterio de Hidalgo. (2020). Hidalgo continuará con semáforo en rojo la próxima semana. Pachuca, Hidalgo: Periódico Criterio de Hidalgo.

[4] Hernández, E. (2020). Sector de la construcción en Hidalgo registra caída de $8.2 \%$. Pachuca, Hidalgo: Milenio Diario.

[5] Hernández, E. (julio de 2020). Ventas han caído en $80 \%$ por covid-19, se espera recuperación económica para noviembre. Pachuca, Hidalgo: Milenio Diario

[6] Gámez, A. (2020). Balnearios de Hidalgo piden que les den fecha de reapertura. Pachuca, Hidalgo.: Periódico Criterio de Hidalgo

[7] Flores, M. (2020). Desempleados durante la cuarentena en Hidalgo llenarían la plaza de toros. Pachuca, Hidalgo: Periódico AM.

[8] Schiffman, L. (2010). El comportamiento del consumidor. Pearson.

[9] Sánchez Garcés, D. (2016). Comportamiento del consumidor en la búsqueda de información de precios on-line.

[10] Torreblanca Diaz, et al. (2012). Comportamiento del consumidor: Cosas ordinarias que se convierten en extraordinarias. 3C Empresa, Investigación y pensamiento crítico.

[11] Brue, S., y Grant, R. (2009). Historia del pensamiento económico. CENGAGE Learning..

[12] Hall, Robert, and Marc Lieberman.(2012) Macroeconomics. Cenage,

[13] Bryant, Keith W. (199) The Economic Organization of the Household, Cambridge, Reino Unido.: Cambridge University Press

[14] Kotler y Keller (2016) Dirección de Mercadotecnia. 15av. Editorial Pearson

[15] Colvin, M. \& Rutland, F. (2008). Is Maslow's Hierarchy of Needs a Valid Model of Motivation. Louisiana Tech University. http://www.business.latech.edu/

[16] Hilgard Ernest y Bower Gordon H (1980) Teorías del Aprendizaje, Trillas, México, 1980.

[17]Skinner, B. F. (1975). La naturaleza genérica de los conceptos de estímulo y respuesta. Barcelona: Fontanella.

[18]Barullas, J. (20 de 12 de 2016). El comportamiento del consumidor y las nuevas tendencias de consumo ante las TIC. https://economiaempresa.blogs.uoc.edu:http://economia-empresa.blogs.uoc.edu/
[19] Sheth, J. (2020a.). Impact of COVID-19 on consumer behavior: Will the old habits return or die? Journal of Business Research, 280-283. doi: https://www.doi.org/10.1016/j.jbusres.2020.05.059

[20]. Vega Barrios A., Cerón Islas A. \& Figueroa Velázquez J.G. (2021) Perspectivas de eCommerce y los Hábitos de Consumo Tras COVID19. European Scientific Journal, ESJ, 17(4), 112. https://doi.org/10.19044/esj.2021.v17n4p112

[21]. López, A. Elizabeth (2021) Pandemia del COVID 19 transformo los hábitos de los consumidores en línea: AMVO. Périodico El Economista. Empresas. Comercio Electrónico. https://www.eleconomista.com.mx/empresas/Compras-en-lineaaumentaron-81-durante-pandemia-en-Mexico-AMVO-202101270077.html

[22]. Comscore MMX, Custom Reporting, Comportamiento de las diferentes categorías durante la pandemia febrero-abril 2020, México.

[23]. Quadratin Hidalgo. (10 de marzo de 2020). Quadratin Hidalgo. https://hidalgo.quadratin.com.mx/principal/hidalgo-sin-casos-de-covid19-activan-plan-de-contingencia-anuncia-fayad/

[24]. La Silla Rota. (17 de marzo de 2020). La Silla Rota Hidalgo Estados Obtenido de Estas son las acciones que se realizan en Operativo Escudo Hidalgo: https://hidalgo.lasillarota.com/acciones-realiza-operativoescudo-en-hidalgo/371218

[25]. Gobierno de México. (17 de mayo de 2020). Gobierno de México, Comisión Ambiental de la Megalopolis. Obtenido de Hoy No Circula en Hidalgo durante la fase 3 de la emergencia sanitaria: https://www.gob.mx/comisionambiental/es/articulos/hoy-no-circulaen-hidalgo-durante-la-fase-3-de-la-emergencia-sanitaria242864?idiom $=$ es 